\title{
A Slight Improvement to the Colored Bárány's Theorem
}

\author{
Zilin Jiang 姜子麟* \\ Department of Mathematical Sciences \\ Carnegie Mellon University \\ Pittsburgh, PA, U.S.A. \\ zilinj@andrew. cmu . edu
}

Submitted: May 14, 2014; Accepted: Oct 30, 2014; Published: Nov 20, 2014

Mathematics Subject Classification: 05C35, 05D99, 05C38

\begin{abstract}
Suppose $d+1$ absolutely continuous probability measures $m_{0}, \ldots, m_{d}$ on $\mathbb{R}^{d}$ are given. In this paper, we prove that there exists a point of $\mathbb{R}^{d}$ that belongs to the convex hull of $d+1$ points $v_{0}, \ldots, v_{d}$ with probability at least $\frac{2 d}{(d+1) !(d+1)}$, where each point $v_{i}$ is sampled independently according to probability measure $m_{i}$.
\end{abstract}

\section{Introduction}

Let $P \subset \mathbb{R}^{d}$ be a set of $n$ points. Every $d+1$ of them span a simplex, for a total of $\left(\begin{array}{c}n \\ d+1\end{array}\right)$ simplices. The point selection problem asks for a point contained in as many simplices as possible. Boros and Füredi [BF84] showed for $d=2$ that there always exists a point in $\mathbb{R}^{2}$ contained in at least $\frac{2}{9}\left(\begin{array}{l}n \\ 3\end{array}\right)-O\left(n^{2}\right)$ simplices. A short and clever proof of this result was given by Bukh [Buk06]. Bárány [Bár82] generalized this result to higher dimensions:

Theorem 1 (Bárány [Bár82]). There exists a point in $\mathbb{R}^{d}$ that is contained in at least $c_{d}\left(\begin{array}{c}n \\ d+1\end{array}\right)-O\left(n^{d}\right)$ simplices, where $c_{d}>0$ is a constant depending only on the dimension $d$.

This general result, the Bárány's theorem, is also known as the first selection lemma. We will henceforth denote by $c_{d}$ the largest possible constant for which the Bárány's theorem holds true. Bukh, Matoušek and Nivasch [BMN10] used a specific construction called the stretched grid to prove that the constant $c_{2}=\frac{2}{9}$ in the planar case found by Boros and Füredi [BF84] is the best possible. In fact, they proved that $c_{d} \leqslant \frac{d !}{(d+1)^{d}}$. On the other hand, Bárány's proof in [Bár82] implies that $c_{d} \geqslant(d+1)^{-d}$, and Wagner [Wag03] improved it to $c_{d} \geqslant \frac{d^{2}+1}{(d+1)^{d+1}}$.

\footnotetext{
*Supported in part by U.S. taxpayers through NSF grants DMS-1201380 and DMS-1301548.
} 


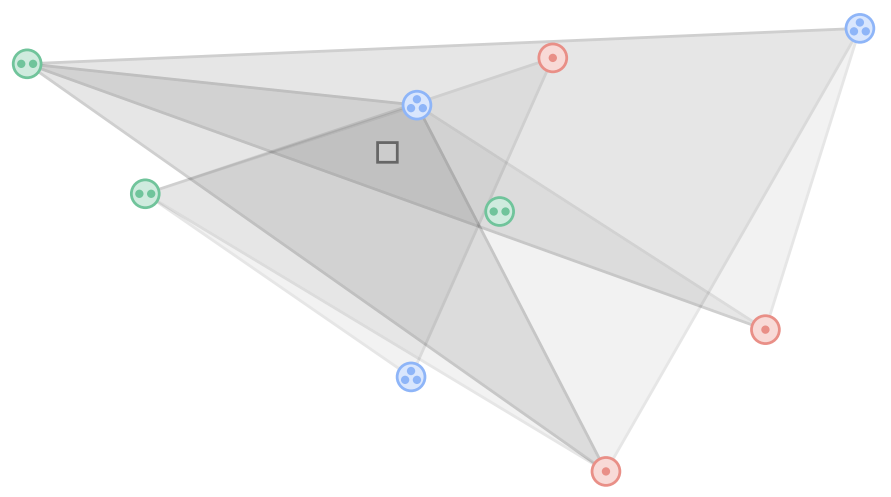

Figure 1: 3 red points, 3 green points and 3 blue points are placed in the plane. The point marked by a square is contained in $6\left(=\frac{2}{9} \cdot 3^{3}\right)$ colorful triangles.

Gromov [Gro10] further improved the lower bound on $c_{d}$ by topological means. His method gives $c_{d} \geqslant \frac{2 d}{(d+1)(d+1) !}$. Matoušek and Wagner [MW11] provided an exposition of the combinatorial component of Gromov's approach in a combinatorial language, while Karasev [Kar12] found a very elegant proof of Gromov's bound, which he described as a "decoded and refined" version of Gromov's proof.

The exact value of $c_{d}$ has been the subject of ongoing research and is unknown, except for the planar case. Basit, Mustafa, Ray and Raza [BMRR10] and successively Matoušek and Wagner [MW11] improved the Bárány's theorem in $\mathbb{R}^{3}$. Král', Mach and Sereni [KMS12] used flag algebras from extremal combinatorics and managed to further improve the lower bound on $c_{3}$ to more than 0.07480 , whereas the best upper bound known is 0.09375 .

However, in this paper, we are concerned with a colored variant of the point selection problem. Let $P_{0}, \ldots, P_{d}$ be $d+1$ disjoint finite sets in $\mathbb{R}^{d}$. A colorful simplex is the convex hull of $d+1$ points each of which comes from a distinct $P_{i}$. For the colored point selection problem, we are concerned with the point(s) contained in many colorful simplices. Karasev proved:

Theorem 2 (Karasev [Kar12]). Given a family of $d+1$ absolutely continuous probability measures $\mathbf{m}=\left(m_{0}, \ldots, m_{d}\right)$ on $\mathbb{R}^{d}$, an $\mathbf{m}$-simplex ${ }^{1}$ is the convex hull of $d+1$ points $v_{0}, \ldots, v_{d}$ with each point $v_{i}$ sampled independently according to probability measure $m_{i}$. There exists a point of $\mathbb{R}^{d}$ that is contained in an $\mathbf{m}$-simplex with probability $p_{d} \geqslant \frac{1}{(d+1) !}$. In addition, if two probability measures coincide, then the probability can be improved to $p_{d} \geqslant \frac{2 d}{(d+1)(d+1) !}$.

By a standard argument which we will provide immediately, a result on the colored point selection problem follows:

Corollary 3. If $P_{0}, \ldots, P_{d}$ each contains $n$ points, then there exists a point that is contained in at least $\frac{1}{(d+1) !} \cdot n^{d+1}$ colorful simplices.

\footnotetext{
${ }^{1} \mathrm{An} \mathbf{m}$-simplex is actually a simplex-valued random variable.
} 
Our result drops the additional assumption in theorem 2, hence improves corollary 3 :

Main Theorem. There is a point in $\mathbb{R}^{d}$ that belongs to an $\mathbf{m}$-simplex with probability $p_{d} \geqslant \frac{2 d}{(d+1)(d+1) !}$.

Corollary 4. There exists a point that is contained in at least $\frac{2 d}{(d+1)(d+1) !} \cdot n^{d+1}$ colorful simplices.

Proof of corollary 4 from the main theorem. Given $d+1$ sets $P_{0}, \ldots, P_{d}$ in $\mathbb{R}^{d}$ each of which contains $n$ points. Let $\Psi: \mathbb{R}^{d} \rightarrow \mathbb{R}$ be the bump function defined by $\Psi\left(x_{1}, \ldots, x_{d}\right)=$ $\prod_{i=1}^{d} \psi\left(x_{i}\right)$, where $\psi(x)=e^{-1 /\left(1-x^{2}\right)} \mathbf{1}_{|x|<1}$, and set $\Psi_{n}\left(x_{1}, \ldots, x_{d}\right)=n^{d} \Psi\left(n x_{1}, \ldots, n x_{d}\right)$ for $n \in \mathbb{N}$. It is a standard fact that $\Psi$ and $\Psi_{n}$ are absolutely continuous probability measures supported on $[-1,1]^{d}$ and $[-1 / n, 1 / n]^{d}$ respectively.

For each $n \in \mathbb{N}$ and $0 \leqslant k \leqslant d$, define $m_{k}^{(n)}(x):=\frac{1}{n} \sum_{p \in P_{k}} \Psi_{n}(x-p)$ for $x \in$ $\mathbb{R}^{d}$. Note that $m_{k}^{(n)}$ is an absolutely continuous probability measure supported on the Minkowski sum of $P_{k}$ and $[-1 / n, 1 / n]^{d}$. Let $\mathbf{m}^{(n)}$ be the family of $d+1$ probability measures $m_{0}^{(n)}, \ldots, m_{d}^{(n)}$. By the main theorem, there is a point $p^{(n)}$ of $\mathbb{R}^{d}$ that belongs to an $\mathbf{m}^{(n)}$-simplex with probability at least $\frac{2 d}{(d+1)(d+1) !}$.

Because no point in a certain neighborhood of infinity is contained in any $\mathbf{m}^{(n)}$-simplex, the set $\left\{p^{(n)}: n \in \mathbb{N}\right\}$ is bounded, and consequently the set has a limit point $p$. Suppose $p$ is contained in $N$ colorful simplices. Let $\epsilon>0$ be the distance from $p$ to all the colorful simplices that do not contain $p$. Choose $n$ large enough such that $1 / n \ll \epsilon$ and $\left|p^{(n)}-p\right| \ll$ $\epsilon$. By the choice of $n$, if $p$ is not contained in a colorful simplex spanned by $v_{0}, \ldots, v_{d}$, then $p^{(n)}$ is not contained the convex hull of $v_{0}^{\prime}, \ldots, v_{d}^{\prime}$ for all $v_{i}^{\prime} \in v_{i}+[-1 / n, 1 / n]^{d}$. This implies that the probability that $p^{(n)}$ is contained in an $\mathbf{m}^{(n)}$-simplex is at most $\frac{N}{n^{d+1}}$. Hence $p$ is the desired point contained in $N \geqslant \frac{2 d}{(d+1)(d+1) !} \cdot n^{d+1}$ colorful simplices.

Readers who are familiar with Karasev's work [Kar12] would notice that our proof of the main theorem heavily relies on his arguments. The author is deeply in debt to him.

\section{Proof of the Main Theorem}

In this section, we provide the proof of the main theorem. The topological terms in the proof are standard, and can be found in [Mat03]. In addition to the notion of an $\mathbf{m}$ simplex, in the proof, we will often refer to an $\left(m_{k}, \ldots, m_{d}\right)$-face which means the convex hull of $d-k+1$ points $v_{k}, \ldots, v_{d}$ with each point $v_{i}$ sampled independently according to probability measure $m_{i}$. An $\mathbf{m}$-simplex and an $\left(m_{k}, \ldots, m_{d}\right)$-face are both set-valued random variables.

Proof of the main theorem. To obtain a contradiction, we suppose that for any point $v$ in $\mathbb{R}^{d}$, the probability that $v$ belongs to an $\mathbf{m}$-simplex is less than $p_{d}:=\frac{2 d}{(d+1)(d+1) !}$. Since this probability, as a function of point $v$, is continuous and uniformly tends to 0 as $v$ goes to infinity, there is an $\epsilon>0$ such that $v$ is contained in an $\mathbf{m}$-simples with probability at most $p_{d}-\epsilon$ for all $v$ in $\mathbb{R}^{d}$. 

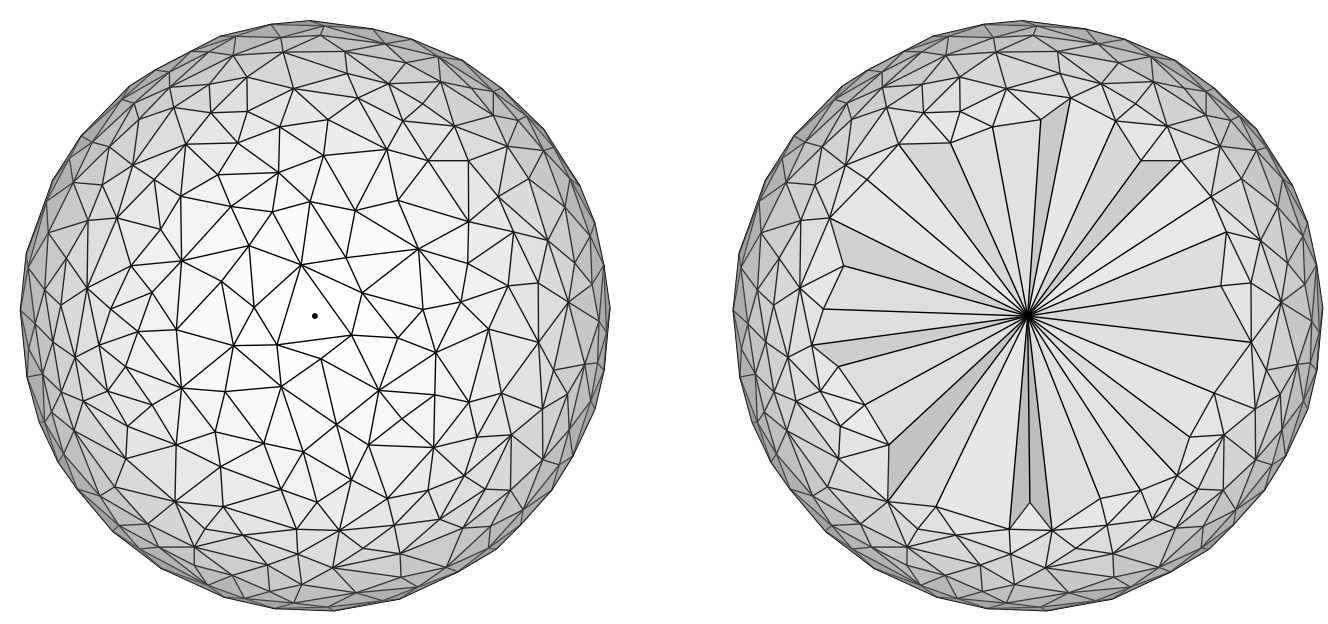

Figure 2: The bird's-eye view of a triangulation of $S^{2}$ with a 2 -simplex containing $\infty$ and the cone over part of the triangulation.

Let $S^{d}:=\mathbb{R}^{d} \cup\{\infty\}$ be the one-point compactification of the Euclidean space $\mathbb{R}^{d}$. Take $\delta=\epsilon / d$. Choose a finite triangulation ${ }^{2} \mathcal{T}$ of $S^{d}$ with one of the $d$-simplices containing $\infty$ such that for $0<k \leqslant d$, any $k$-face of $\mathcal{T}$ intersects an $\left(m_{k}, \ldots, m_{d}\right)$-face with probability less than $\delta$ and that the measure of any $d$-face of $\mathcal{T}$ under $\left(m_{d-1}+m_{d}\right) / 2$ is less than $\delta$. This can be done by taking a sufficiently fine triangulation of $S^{2}$ with one $d$-simplex having $\infty$ in its relative interior.

We use cone $(\cdot)$ as the cone functor ${ }^{3}$ with apex $O$. A triangulation $\mathcal{T}$ of $S^{d}$ naturally extends to a triangulation $\operatorname{cone}(\mathcal{T})$ of $\operatorname{cone}\left(S^{d}\right)$. We denote the $k$-skeleton ${ }^{4}$ of $\mathcal{T}$ and cone $(\mathcal{T})$ by $\mathcal{T} \leqslant k$ and cone $(\mathcal{T})^{\leqslant k}$ respectively.

We are going to define a continuous map $f: \operatorname{cone}(\mathcal{T})^{\leqslant d} \rightarrow S^{d}$. Put $f(x)=x$ for all $x \in S^{d}=\|\mathcal{T}\| \subset\left\|\operatorname{cone}(\mathcal{T})^{\leqslant d}\right\|$, and set $f(O)=\infty$. We proceed to define $f$ on $\operatorname{cone}(\sigma)$ for all the $k$-faces $\sigma$ of $\mathcal{T}$ inductively on dimension $k$ of $\sigma$ while we maintain the property that the image of the boundary of cone $(\sigma)$ under $f$, that is $f(\partial \operatorname{cone}(\sigma))$, intersects an $\left(m_{k}, \ldots, m_{d}\right)$-face with probability at most $(k+1) !\left(p_{d}-\epsilon+k \delta\right)$. We say $f$ is economical over a $k$-face $\sigma$ of $\mathcal{T}^{\leqslant d-1}$ if $f$ and $\sigma$ satisfy the above property. Unlike Karasev [Kar12], our inductive construction of $f$ follows the same pattern until $k=d-2$ instead of $d-1$. The main innovation of this proof is a different construction for $k=d-1$, which enables us to remove the additional assumption in theorem 2 .

Note that for any 0 -face $\sigma$ in $\mathcal{T}, f(\partial \operatorname{cone}(\sigma))=f(\{\sigma, O\})=\{\sigma, \infty\}$. According to the assumption at the beginning of the proof, $f(\partial \operatorname{cone}(\sigma))$ intersects an $\left(m_{0}, \ldots, m_{d}\right)$-face,

\footnotetext{
${ }^{2}$ A triangulation $\mathcal{T}$ of a topological space $X$ is a simplicial complex $\mathrm{K}$, homeomorphic to $X$, together with a homeomorphism $h:\|\mathrm{K}\| \rightarrow X$. Since the finite triangulation of interest is an extension of the triangulation of a $d$-simplex $X$ in $\mathbb{R}^{d}$ and $h$ is an identity map, we will freely use topological notions such as "a $k$-face (as a subset of $S^{d}$ )" instead of "the image of a $k$-face in K under $h$ ". With such abuse of language, we can avoid going back and forth between the simplicial complex and the topological space.

${ }^{3}$ The cone over a space $X$ is the quotient space cone $(X):=(X \times[0,1]) /(X \times\{1\})$. The apex is the equivalence class $\{(x, 1): x \in X\}$.

${ }^{4}$ The $k$-skeleton of a simplicial complex $\Delta$ consists of all simplices of $\Delta$ of dimension at most $k$.
} 
that is, an $\mathbf{m}$-simplex, with probability at most $p_{d}-\epsilon$. Therefore $f$ is economical over 0 -faces of $\mathcal{T}$. This finishes the first step.

Suppose $f$ is already defined on $\operatorname{cone}(\mathcal{T})^{\leqslant k}$ and it is economical over $k$-faces of $\mathcal{T}$. We are going to extend the domain of $f$ to $\operatorname{cone}(\mathcal{T})^{\leqslant k+1}$. Indeed, we only need to define $f$ on cone $(\sigma)$ for every $k$-face $\sigma$ of $\mathcal{T}$.

Take any $k$-face $\sigma$ of $\mathcal{T}$. Suppose convex hull of $v_{k}, \ldots, v_{d}$, denoted by $\operatorname{conv}\left(v_{k}, \ldots, v_{d}\right)$, is an $\left(m_{k}, \ldots, m_{d}\right)$-face. Notice that the following statements are equivalent:

- $f(\partial \operatorname{cone}(\sigma))$ intersects $\operatorname{conv}\left(v_{k}, \ldots, v_{d}\right)$;

- for some $v \in f(\partial \operatorname{cone}(\sigma))$, the ray with initial point $v$ in the direction $\overrightarrow{v_{k} v}$ intersects $\operatorname{conv}\left(v_{k+1}, \ldots, v_{d}\right)$.

We call the union of such rays the shadow of $f(\partial \operatorname{cone}(\sigma))$ centered at $v_{k}$. Since $f$ is economical over $\sigma$, the probability for an $\left(m_{k}, \ldots, m_{d}\right)$-face to meet $f(\partial \operatorname{cone}(\sigma))$ is at most $(k+1) !\left(p_{d}-\epsilon+k \delta\right)$, and so there exists $v_{k}^{\sigma} \in \mathbb{R}^{d}$ such that the shadow of $f(\partial \operatorname{cone}(\sigma))$ centered at $v_{k}^{\sigma}$ intersects $\operatorname{conv}\left(v_{k+1}, \ldots, v_{d}\right)$ with probability at most $(k+1) !\left(p_{d}-\epsilon+k \delta\right)$.

Now, we define $f$ on cone $(\sigma)$. First, let $g$ be the homeomorphism from cone $(\sigma)$ onto the cone over $\partial \operatorname{cone}(\sigma)$ with apex $c$ such that $g$ is an identity on $\partial \operatorname{cone}(\sigma)$. This can be done because cone $(\sigma)$ is homeomorphic to a $(k+1)$-simplex $\Delta$ and it is easy to find a homeomorphism from $\Delta$ to cone $(\partial \Delta)$ that keeps $\partial \Delta$ fixed.
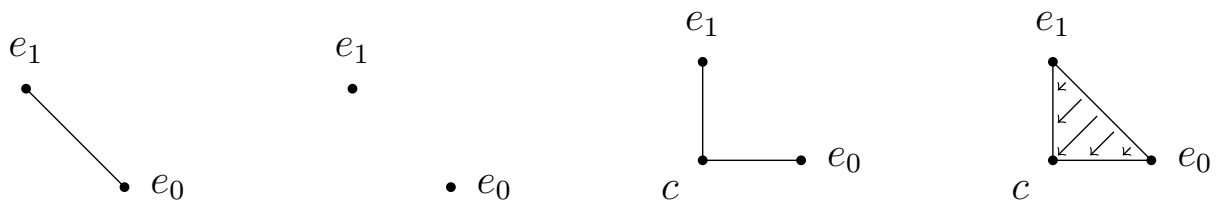

Figure 3: An illustration of an 1-simplex $\Delta, \partial \Delta$, cone $(\partial \Delta)$ and a homeomorphism from $\Delta$ to cone $(\partial \Delta)$.

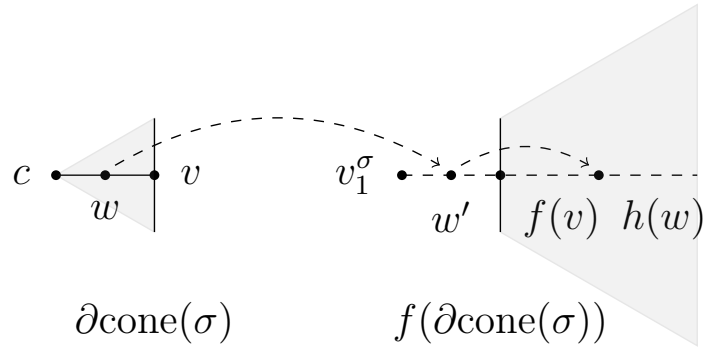

Figure 4: The illustration shows a cone over part of $\partial \operatorname{cone}(\sigma)$ with apex $c$ and a point $v$ on the boundary, and how a point $w$ on the line segment $[v, c)$ are mapped under $h$.

Next, note that every point $w$ in $\operatorname{cone}(\sigma)$ except $c$ is on a line segment $[v, c)$ for a unique point $v$ on $\partial \operatorname{cone}(\sigma)$. If $t=\overline{v w} / \overline{w c} \in[0, \infty)$, then put $h(w)=\overrightarrow{f(v)}+t \cdot \overrightarrow{v_{k}^{\sigma} f(v)}$. In 
addition, set $h(c)=\infty$. The function $h$ maps $[v, c)$ onto $\left[f(v), v_{k}^{\sigma}\right)$ linearly and then takes the inversion centered at $v_{k}^{\sigma}$ with radius $\overline{v_{k}^{\sigma} f(v)}$ so that $\left[f(v), v_{k}^{\sigma}\right)$ gets mapped onto the

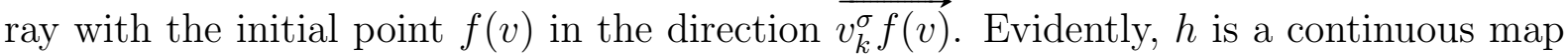
from cone $(\partial \operatorname{cone}(\sigma))$ onto the shadow of $f(\partial \operatorname{cone})$ centered at $v_{k}^{\sigma}$ that coincides with $f$ on $\partial \operatorname{cone}(\sigma)$.

Define $f$ on cone $(\sigma)$ to be the composition of $g$ and $h$ :

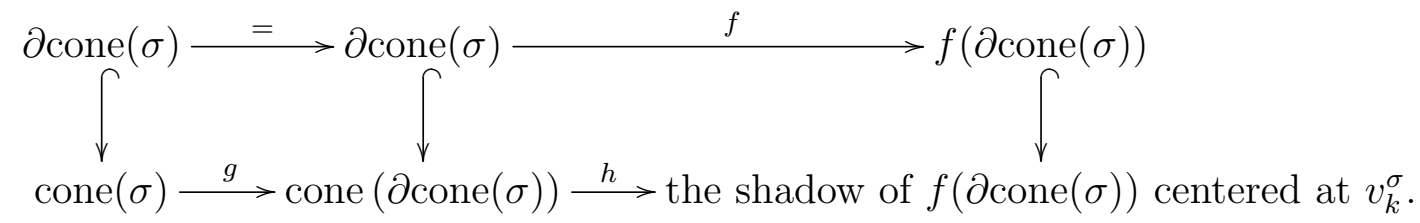

According to the commutative diagram above, $f$ is well-defined on cone $(\sigma)$ in the sense that it is compatible with its definition on $\operatorname{cone}(\mathcal{T})^{\leqslant k}$. We use the phrase "fill in the boundary of cone $(\sigma)$ against the center $v_{k}^{\sigma}$ " to represent the above process that extends the domain of $f$ from $\partial \operatorname{cone}(\sigma)$ to cone $(\sigma)$.

To complete the inductive step, we must demonstrate that $f$ is economical over $(k+1)$ faces of $\mathcal{T}$. Pick any $(k+1)$-face $\tau$ of $\mathcal{T}$. Let $\sigma_{0}, \ldots, \sigma_{k+1}$ be the $k$-faces of $\tau$. Observing that $f(\partial \operatorname{cone}(\tau))=f(\tau \cup \operatorname{cone}(\partial \tau))=\tau \cup f\left(\operatorname{cone}\left(\sigma_{0}\right)\right) \cup \ldots \cup f\left(\operatorname{cone}\left(\sigma_{k+1}\right)\right)$ and that $f\left(\operatorname{cone}\left(\sigma_{i}\right)\right)$ is the shadow of $f\left(\partial \operatorname{cone}\left(\sigma_{i}\right)\right)$ centered at $v_{k}^{\sigma_{i}}$ which intersects an $\left(m_{k+1}, \ldots, m_{d}\right)$-face with probability at most $(k+1) !\left(p_{d}-\epsilon+k \delta\right)$, we obtain that the probability for an $\left(m_{k+1}, \ldots, m_{d}\right)$-face to intersect $f(\partial \operatorname{cone}(\tau))$ is dominated by $\delta+(k+2)(k+1) !\left(p_{d}-\epsilon+\right.$ $k \delta) \leqslant(k+2) !\left(p_{d}-\epsilon+(k+1) \delta\right)$.

We have so far defined a continuous map $f$ on cone $(\mathcal{T})^{\leqslant d-1}$ such that for any $(d-1)$ face $\sigma$ of $\mathcal{T}$ the probability for an $\left(m_{d-1} m_{d}\right)$-face to intersect $D:=f(\partial \operatorname{cone}(\sigma))$ is at most $d !\left(p_{d}-\epsilon+(d-1) \delta\right)$. We write $f(X) \bmod 2:=\left\{y \in f(X):\left|f^{-1}(y) \cap X\right|=1(\bmod 2)\right\}$ for the set of points in $f(X)$ whose fibers in $X$ have an odd number of points. Set $\bar{m}:=\left(m_{d-1}+m_{d}\right) / 2$. We are going to define $f$ on cone $(\sigma)$ such that $\bar{m}(f(\operatorname{cone}(\sigma)) \bmod 2)$ is less than $\frac{1-\delta}{d+1}$.

Fix a point $s$ in $\mathbb{R}^{d} \backslash D$. For any point $t$ in $\mathbb{R}^{d} \backslash D$, if a generic piecewise linear path from $s$ to $t$ intersects with $D$ an odd number of times, then put $t$ in $B$, otherwise put it in $A$. Here the number of intersections of a piecewise linear path $L$ and $D$ might not be the cardinality of $L \cap D$. Instead, the number of intersections is precisely $\sum_{x \in L \cap D}\left|f^{-1}(x) \cap \partial \operatorname{cone}(\sigma)\right|$, that is, it takes the multiplicity into account. Thus we have partitioned $\mathbb{R}^{d} \backslash D$ into $A$ and $B$ such that any generic piecewise linear path from a point in $A$ to a point in $B$ meets $D$ an odd number of times. Suppose $a:=m_{d-1}(A), b:=m_{d}(A)$ and $x:=\bar{m}(A)=(a+b) / 2$. The probability that an $\left(m_{d-1} m_{d}\right)$-face intersects with $D$ is at least $a(1-b)+(1-a) b$. Hence $a(1-b)+(1-a) b<d !\left(p_{d}-\epsilon+(d-1) \delta\right)<2\left(\frac{1-\delta}{d+1}\right)\left(1-\frac{1-\delta}{d+1}\right)$. Because $a(1-b)+(1-a) b=$ $(a+b)-2 a b \geqslant(a+b)-(a+b)^{2} / 2=2 x(1-x)$, either $x$ or $1-x$ is less than $\frac{1-\delta}{d+1}$. In other words, one of $\bar{m}(A)$ and $\bar{m}(B)$ is less than $\frac{1-\delta}{d+1}$. We may assume that $\bar{m}(B)<\frac{1-\delta}{d+1}$.

Fix a point $c \in A$. Again, we fill in the boundary of cone $(\sigma)$ against the center $c$. For any generic point $x \in A$, the line segment $[c, x]$ intersects with $D$ an even number of times. For every $v$ on $\partial \operatorname{cone}(\sigma)$, the ray with the initial point $f(v)$ in the direction $\overrightarrow{c f(v)}$ 

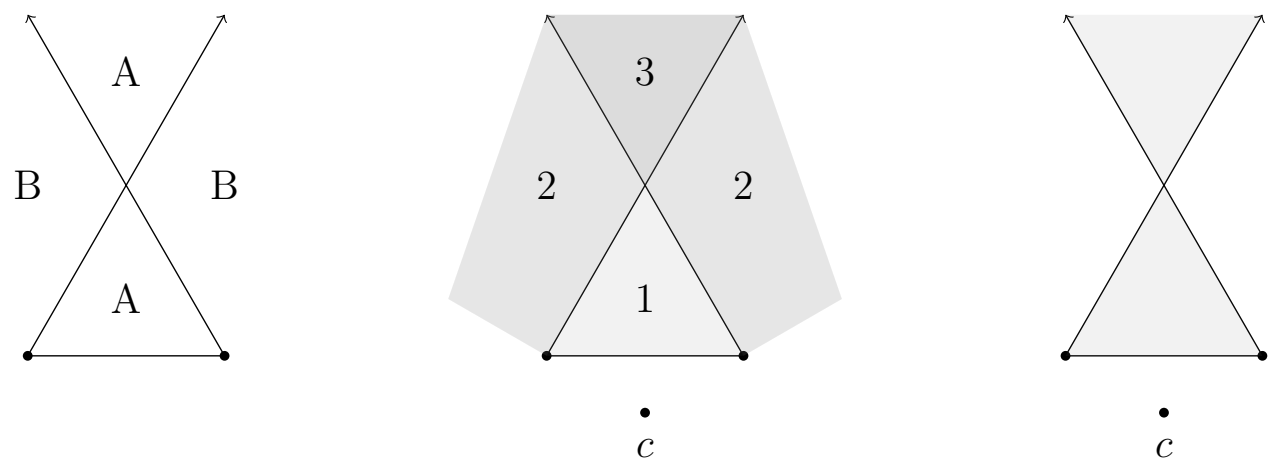

Figure 5: An illustration of the partition, the result of filling in against $c$, and $f(\operatorname{cone}(\sigma)) \bmod 2$.

covers $x$ once if and only if the line segment $[c, x]$ intersects with $D$ at $f(v)$. Because $f(\operatorname{cone}(\sigma))$ is the union of such rays, the number of times that $x$ is covered by $f(\operatorname{cone}(\sigma))$ is exactly the number of intersections between $[c, x]$ and $D$. This implies that $x$ is not in $f(\operatorname{cone}(\sigma)) \bmod 2$. Therefore $f(\operatorname{cone}(\sigma)) \bmod 2$ is a subset of $B \cup D$ almost surely. Noticing that $\bar{m}(D)=0$, the extension of $f$ has the desired property $\bar{m}(f(\operatorname{cone}(\sigma)) \bmod 2)<\frac{1-\delta}{d+1}$.

Pick any $d$-face $\tau$ of $\mathcal{T}$. Suppose the $(d-1)$-faces of $\tau$ are $\sigma_{0}, \ldots, \sigma_{d}$. By a parity argument, we have

$$
\begin{aligned}
f(\partial \operatorname{cone}(\tau)) \bmod 2 & =\left[\tau \cup f\left(\operatorname{cone}\left(\sigma_{0}\right)\right) \cup \ldots \cup f\left(\operatorname{cone}\left(\sigma_{d}\right)\right)\right] \bmod 2 \\
& \subset \tau \cup f\left(\operatorname{cone}\left(\sigma_{0}\right)\right) \bmod 2 \cup \ldots \cup f\left(\operatorname{cone}\left(\sigma_{d}\right)\right) \bmod 2 .
\end{aligned}
$$

Therefore $\bar{m}(f(\partial \operatorname{cone}(\tau)) \bmod 2)$ is less than $\delta+(d+1) \frac{1-\delta}{d+1}=1$, and so the degree of $f$ on $\partial \operatorname{cone}(\tau)$, denoted by $\operatorname{deg}(f, \partial \operatorname{cone}(\tau))$, is even. Because

$$
\sum_{\tau} \operatorname{deg}(f, \partial \operatorname{cone}(\tau))=2 \sum_{\sigma} \operatorname{deg}(f, \operatorname{cone}(\sigma))+\operatorname{deg}(f, \mathcal{T})=\operatorname{deg}(f, \mathcal{T}) \quad(\bmod 2),
$$

where the first sum and the second sum are over all $d$-faces and all $(d-1)$-faces of $\mathcal{T}$ respectively, we know that $\operatorname{deg}(f, \mathcal{T})$ is even, which contradicts with the fact that $f$ is identity on $\mathcal{T}$.

\section{Acknowledgment}

The author would like to thank Boris Bukh for guidance and fruitful discussions on the Bárány's theorem. This article would not have been possible without his support. The author is also grateful to Roman Karasev and/or the anonymous referee who read the preliminary version of the paper and pointed out many inaccuracies.

\section{References}

[Bár82] Imre Bárány. A generalization of Carathéodory's theorem. Discrete Math., 40(2-3):141-152, 1982. 
[BF84] E. Boros and Z. Füredi. The number of triangles covering the center of an n-set. Geom. Dedicata, 17(1):69-77, 1984.

[BMN10] Boris Bukh, Jiří Matoušek, and Gabriel Nivasch. Stabbing simplices by points and flats. Discrete Comput. Geom., 43(2):321-338, 2010.

[BMRR10] Abdul Basit, Nabil H. Mustafa, Saurabh Ray, and Sarfraz Raza. Improving the first selection lemma in $\mathbb{R}^{3}$. In Computational geometry (SCG'10), pages 354-357. ACM, New York, 2010.

[Buk06] Boris Bukh. A point in many triangles. Electron. J. Combin., 13(1):\#N10, 2006.

[Gro10] Mikhail Gromov. Singularities, expanders and topology of maps. Part 2: From combinatorics to topology via algebraic isoperimetry. Geom. Funct. Anal., 20(2):416-526, 2010.

[Kar12] Roman Karasev. A simpler proof of the Boros-Füredi-Bárány-Pach-Gromov theorem. Discrete Comput. Geom., 47(3):492-495, 2012.

[KMS12] Daniel Král', Lukáš Mach, and Jean-Sébastien Sereni. A new lower bound based on Gromov's method of selecting heavily covered points. Discrete Comput. Geom., 48(2):487-498, 2012.

[Mat03] Jiří Matoušek. Using the Borsuk-Ulam theorem: lectures on topological methods in combinatorics and geometry. Springer, 2003.

[MW11] Jiří Matoušek and Uli Wagner. On Gromov's method of selecting heavily covered points. arXiv preprint arXiv:1102.3515, 2011.

[Wag03] Ulrich Wagner. On k-sets and applications. PhD thesis, Swiss Federal Institute of Technology, ETH Zürich, 2003. 